\title{
High-performance 16-way Ku-band radial power combiner based on the TE01-circular waveguide mode
}

\author{
José R. Montejo-Garai, ${ }^{1}$ Irene O. Saracho-Pantoja, ${ }^{1}$ Jorge A. Ruiz-Cruz, ${ }^{2}$ \\ and Jesús M. Rebollar ${ }^{1}$ \\ ${ }^{1}$ Grupo de Electromagnetismo Aplicado, Information Processing and Telecommunications Center, \\ Universidad Politécnica de Madrid, Madrid, Spain \\ ${ }^{2}$ Escuela Politécnica Superior, Universidad Autónoma de Madrid, Madrid, Spain
}

(Received 10 January 2018; accepted 18 February 2018; published online 7 March 2018)

\begin{abstract}
This work presents a 16-way Ku-band radial power combiner for high power and high frequency applications, using the very low loss $\mathrm{TE}_{01}$ circular waveguide mode. The accomplished design shows an excellent performance: the experimental prototype has a return loss better than $30 \mathrm{~dB}$, with a balance for the amplitudes of $( \pm 0.15 \mathrm{~dB})$ and $\left( \pm 2.5^{\circ}\right)$ for the phases, in a $16.7 \%$ fractional bandwidth $(2 \mathrm{GHz}$ centered at $12 \mathrm{GHz}$ ). For obtaining these outstanding specifications, required, for instance, in highfrequency amplification or on plasma systems, a rigorous step-by-step procedure is presented. First, a high-purity mode transducer has been designed, from the $\mathrm{TE}_{10}$ mode in the rectangular waveguide to the $\mathrm{TE}_{01}$ mode in the circular waveguide, with very high attenuation $(>50 \mathrm{~dB})$ for the other propagating and evanescent modes in the circular waveguide. This transducer has been manufactured and measured in a back-to-back configuration, validating the design process. Second, an E-plane 16-way radial power divider has been designed, where the power is coupled from the 16 non-reduced-height radial standard waveguides into the $\mathrm{TE}_{01}$ circular waveguide mode, improving the insertion loss response and removing the usual tapered transformers of previous designs limiting the power handling. Finally, both the transducer and the divider have been assembled to make the final radial combiner. The prototype has been carefully manufactured, showing very good agreement between the measurements and the full-wave simulations. Published by AIP Publishing. https://doi.org/10.1063/1.5022056
\end{abstract}

\section{INTRODUCTION}

Nowadays, there is an increasing demand for wideband, high power solid state amplifiers operating in the microwave and millimeter wave frequency ranges. Frequently, a single solid-state power amplifier (SSPA) module is not able to supply enough power necessary to combine various modules to achieve the required level. ${ }^{1-3}$ In scientific research areas such as high-energy particle accelerators and plasma heating for controlled thermonuclear fusion, vacuum electronics amplifiers like Traveling Wave Tubes (TWTs), klystrons, and gyrotrons are used and combined. ${ }^{4}$

Among the different configurations, ${ }^{5,6}$ radial combiners exhibit a number of advantages over the corporate or chaintype combiners, mainly when a large number of ports $(\mathrm{N})$ are considered. The reason is that the power leaving the transducer travels in the radial direction to the $\mathrm{N}$-output ports providing an improved amplitude and phase balance because of the intrinsic symmetry of the waveguide arrangement. Moreover, metallic waveguides are the preferred technology for high-frequency systems requiring high power capability, low insertion loss, and rigid mechanical arrangement, ${ }^{7,8}$ features which are crucial for radial combiners implemented in waveguide technology for the high-frequency modules in amplifiers and plasma systems.

Radial waveguide combiners have been widely used. The work in Ref. 9 presents a wideband $60 \mathrm{GHz}$ 16-way power divider with $12 \mathrm{~dB}$ return loss level in a $20 \%$ bandwidth and in Ref. 10 a 19-way isolated radial combiner in $20 \mathrm{GHz}$ with
$12 \mathrm{~dB}$ return loss level in a $24.4 \%$ bandwidth. A 24-way radial combiner in the Ka band with $25 \mathrm{~dB}$ return loss level in a $15 \%$ bandwidth is proposed in Ref. 11. Recently, a 20-way isolated Ka-band with $10 \mathrm{~dB}$ return loss level in a $25 \%$ bandwidth has been reported in Ref. 12. These values are reported in Table I, where some important parameters of the design that will be introduced in this work are also shown. Additional electric and mechanical key features will be also highlighted in Secs. II-IV.

There are two relevant aspects regarding the radial combiners. The first one is the geometry of the rectangular to circular waveguide transducer connected to the divider, a matter directly related to the compactness of the combiner and the attenuation of the higher-modes. The second one is the possibility of including resistive elements to improve the isolation between output ports. In this case, the insertion loss level, the amplitude and phase balance, and the power handling are usually degraded. The decision of including resistive septa depends on the requirements of the amplification system composed by the Monolithic Microwave Integrated Circuits (MMIC) modules and the two combiners. ${ }^{13}$

The goal of this work is to design a compact $\mathrm{Ku}$-band power combiner with high performance, paying special attention to the following key requirements: the return loss level, the insertion loss, the power handling, and the amplitude-phase balance (Fig. 1). In addition, the isolation between output ports will be controlled under suitable level, without including resistive septa, since their advantages for this application in isolation may not be enough to compensate for the more 
TABLE I. Comparison of different radial power combiners based on the $\mathrm{TE}_{01}$ circular waveguide mode.

\begin{tabular}{lcccccc}
\hline \hline Reference & N-way & $\begin{array}{c}\text { Center-frequency } \\
(\mathrm{GHz})\end{array}$ & Bandwidth $(\%)$ & $\begin{array}{c}\text { Return loss } \\
(\mathrm{dB})\end{array}$ & $\begin{array}{c}\text { Mode } \\
\text { transducer }\end{array}$ & Isolation \\
\hline 9 & 16 & 61 & 20 & 12 & Sidewall coupling & No resistive septa \\
10 & 19 & 20.5 & 24.4 & 12 & Flared structure & Resistive septa \\
11 & 24 & 33.5 & 15 & 25 & Flared structure & Resistive septa \\
12 & 20 & 32 & 25 & 10 & Sidewall coupling & Resistive septa \\
This work & 16 & 12 & 16.7 & 30 & Sidewall coupling & No resistive septa \\
\hline \hline
\end{tabular}

complex mounting and the additional insertion loss that they introduce.

\section{DESIGN OF THE TE ${ }_{01}$ MODE TRANSDUCER}

\section{A. Foundation of the mode transducer operation}

The $\mathrm{TE}_{01}$ circular mode transducer is a key device in different fields of microwave engineering. It is of great interest in long transmission systems since the attenuation constant of the $\mathrm{TE}_{01}$ mode, due to finite conductivity walls, is gradually smaller as the frequency increases. ${ }^{15,16}$ Likewise, it is used in the design of very high-Q resonant cavities that are key components in areas such as gyrotrons, plasma-heating systems, maser-based devices, and other fields related with high-energy environments. ${ }^{17-20}$ Furthermore, and really relevant to this work, radial power combiners have extensively used this kind of transducer for long time. ${ }^{10,11}$

There are basically two methods to excite the circular $\mathrm{TE}_{01}$ mode. The first one, according to its historical development, is based on a slowly flared structure to adjust the input rectangular waveguide to the output circular waveguide. Several sections are cascaded in-line under a symmetric arrangement in order to avoid as much as possible the higher order mode generation. As a result of the lack of computer-aided design (CAD) tools to perform the electromagnetic analysis, ${ }^{15}$ this type of converters was designed heuristically until recent time. Some suitable referenced examples are the Southworth-type converter, ${ }^{15,21}$ the Marie-type converter, ${ }^{22,23}$ and the sector converter. ${ }^{24}$ The main drawbacks of this kind of in-line configuration are the great length of the final device and the high level of the excited undesired modes. ${ }^{13}$

The second method uses a sidewall coupling between the rectangular and circular waveguides. ${ }^{25}$ Within this classification, the flower-petal converter can be cited. ${ }^{26}$ Its drawback is double, on the one hand the narrow bandwidth response and on the other hand the high insertion loss level. Transducers in the Ka-band based on routing with four branches are presented in Refs. 12 and 27, showing moderate return loss level. In the above references, the sidewall coupling is just an aperture with limited degrees of freedom. In Ref. 14, the sidewall coupling is improved to enhance the response in broadband and to increase the return loss level.

The transducer based on the sidewall coupling is composed by the converting section, i.e., the structure accomplishing the interaction between the $\mathrm{TE}_{10}$ rectangular mode and the $\mathrm{TE}_{01}$ circular mode, and the feeding network routing the four arms to the input (Fig. 2). A systematic process will be presented to carry out its design to obtain a high performance in both, the return loss and the attenuation of the non-desired modes in the circular waveguide (especially those that are propagating). This is an aspect that is sometimes not reported in the literature with the required detail since the control of the modes is the key to have a successful design and to fulfill the challenging specifications presented in this contribution.

Previously to any other consideration, it must be taken into account that the $\mathrm{TE}_{01}$ mode is not the dominant mode of the circular waveguide. For this reason, it is crucial to analyze the propagating modes considering the number of symmetry planes $(1,2$, or 4$)$ of the physical structure, the excitation (rectangular waveguide $\mathrm{TE}_{10}$ mode), and the desired circular waveguide $\mathrm{TE}_{01}$ mode. Table II shows the modes associated to the cases of one, two, or four symmetry planes and the normalized cut-off frequencies of the modes involved in the structure. In this case, $\mathrm{TE}_{01}$ has the electric wall (EW)

\section{$\mathrm{TE}_{10}$ rectangular waveguide (WR75)}

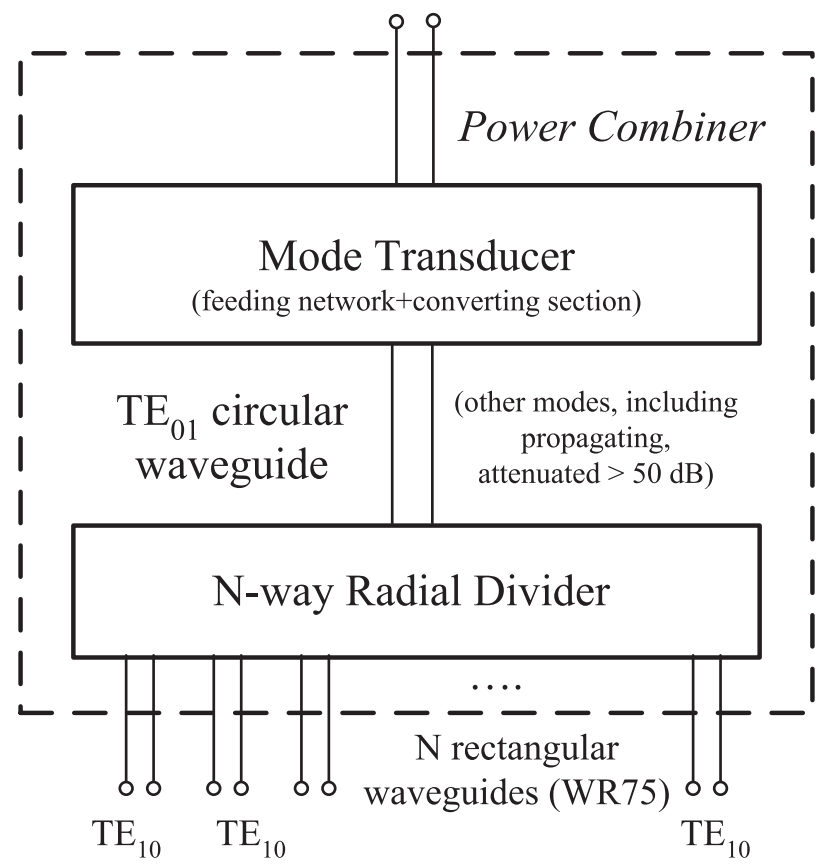

FIG. 1. Scheme of the power combiner composed of the mode transducer between the rectangular mode $\mathrm{TE}_{10}$ and the circular mode $\mathrm{TE}_{01}$, and the $\mathrm{N}$-way radial divider. 


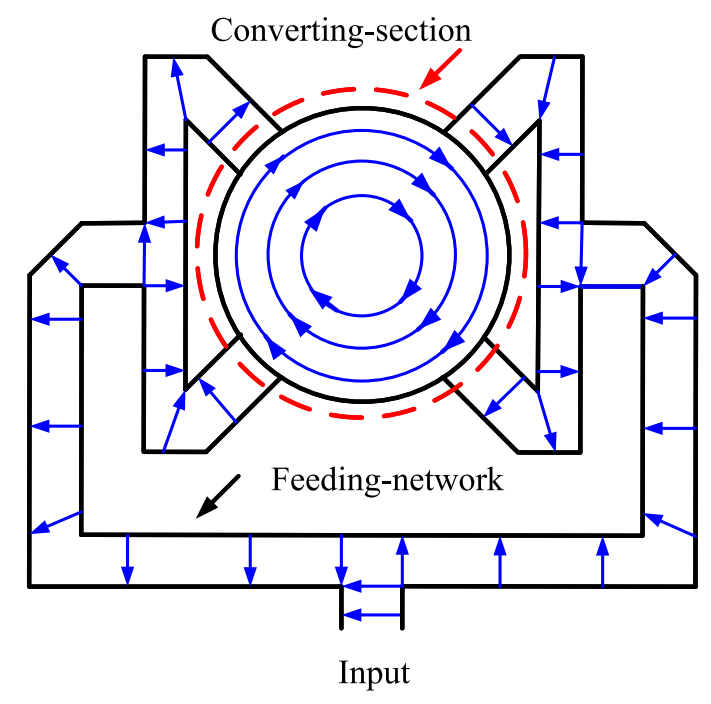

FIG. 2. Scheme of a sidewall coupling $\mathrm{TE}_{01}$-circular mode transducer which consists of the converting-section (dashed circle) and the input feeding network, showing the electric field lines.

boundary condition at four symmetry planes of the circular waveguide including the four excitation ports. Therefore, the following considerations are derived:

(a) Since the converting-section has four symmetry planes with the EW boundary condition in all of them, including the excitation, the only propagating mode in the circular waveguide is $\mathrm{TE}_{01}$ (see the third column of Table II).

(b) The feeding-network has only one symmetry plane.

(c) Thus, the complete transducer has only one symmetry plane with EW symmetry. Therefore, in the optimization of the final transducer, the amplitudes of the propagating modes $\mathrm{TE}_{11 \mathrm{c}}$ and $\mathrm{TE}_{21 \mathrm{c}}$ must be kept under very low levels. Modes $\mathrm{TM}_{11 \mathrm{~s}}, \mathrm{TE}_{31 \mathrm{c}}$, and $\mathrm{TM}_{21 \mathrm{~s}}$ also have to be controlled for avoiding higher-order mode interactions with the radial divider and, thus, allowing to reduce later the connection length between the transducer and the radial divider.

\section{B. Converting-section design}

The first task in the converting-section design is to establish that the excitation will have two symmetry planes (four ports) in order to avoid the generation of the $\mathrm{TE}_{21 \mathrm{c}}$ mode, as said before (see Table II column 3). In addition, some kind of a matching element must be included in order to obtain the desired return loss level in broadband. For this purpose, (see the inset in Fig. 3) a one-section stepped transformer connects the circular and rectangular waveguides. A circular post has also been placed on the circular bottom with the goal of improving the bandwidth and the return loss.

Figure 3 shows the simulated response of the convertingsection, obtained with Computer Simulation Technology $(\mathrm{CST})^{28}$ considering the four symmetry planes in the analysis. The return loss level for the $\mathrm{TE}_{01}$ mode is higher than $30 \mathrm{~dB}$. In the inset, a $3 \mathrm{D}$ CAD view of the full converting-section is included.

\section{Feeding network design}

The next step is to feed the converting-section from the input rectangular port, maintaining the symmetry plane when exciting the four ports (Fig. 2). In this stage, it is necessary to preserve the bandwidth and the return loss level obtained previously in the converting-section, and at the same time, minimizing the total external dimension.

TABLE II. Circular waveguide modes associated to the case of one, two, or four symmetry planes and their normalized cut-off frequencies. In boldface, the circular mode $\mathrm{TE}_{01}$ is used to connect the mode transducer to the $\mathrm{N}$-way radial divider.

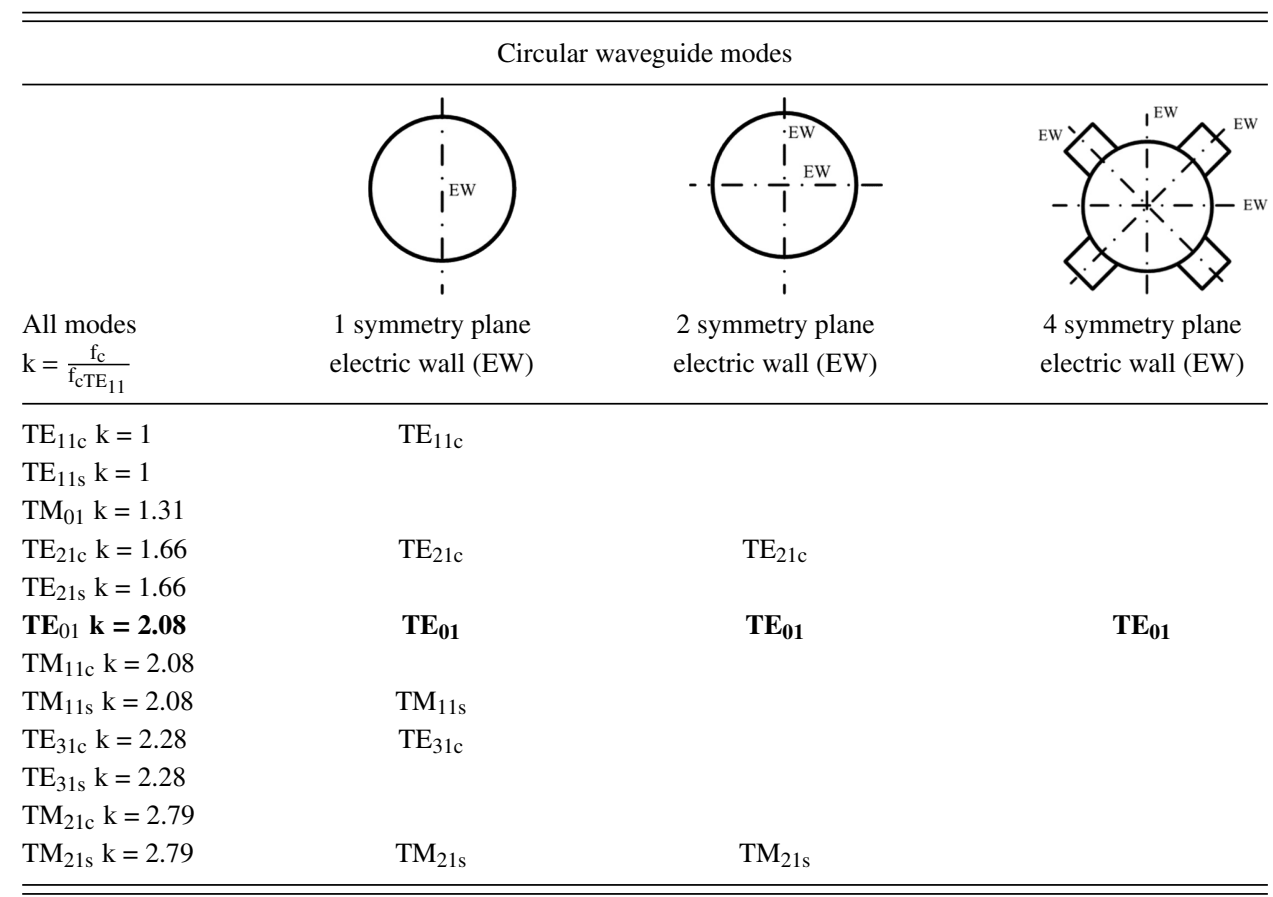




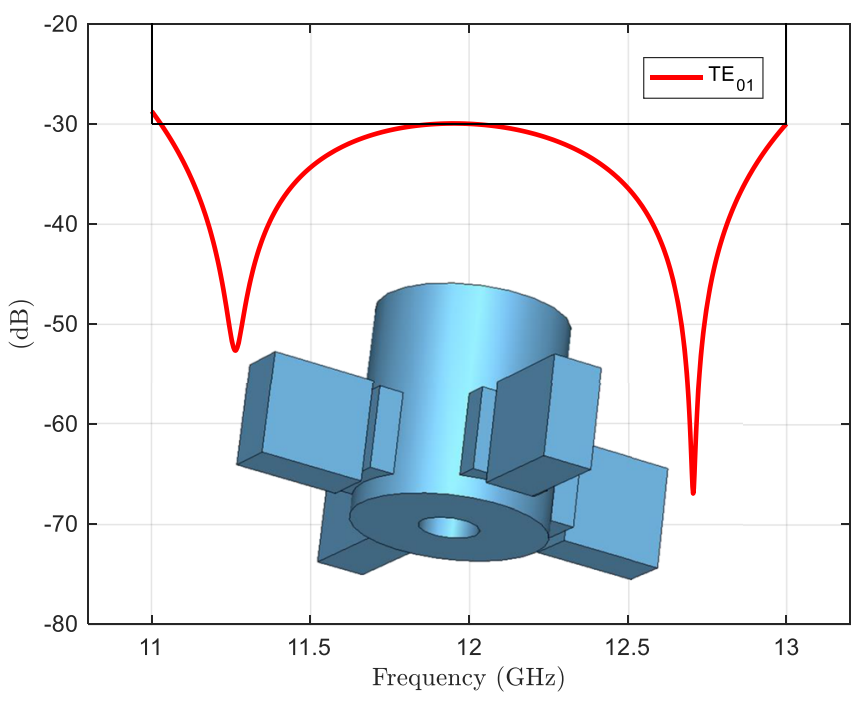

FIG. 3. Simulated response of the reflection coefficient for the $\mathrm{TE}_{01}$ mode of the converting-section, taking into account the four symmetry planes with the electric perfect boundary condition. In the inset, a 3D CAD view of the full converting-section is shown.

The feeding-network is composed of two types of T junctions and three kinds of waveguide bends, all in the E-plane configuration, setting one symmetry plane for the whole structure (see the inset in Fig. 4). A step-by-step process has been carried out in the design of all these individual components separately before a final optimization of the complete feeding structure. For the individual elements, simulations are done considering only one half of the structure with the EW boundary condition in the symmetry plane.

\section{Final design of the transducer and back-to-back measurement}

Following the sequential procedure, the two parts (converting section and feeding network) individually designed

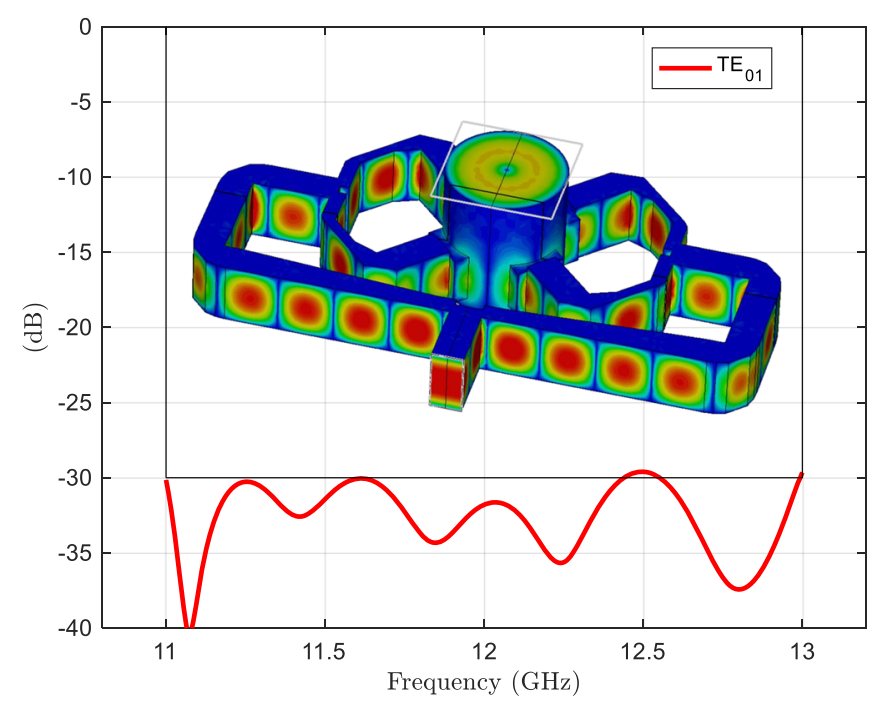

FIG. 4. Simulated return loss of the transducer, higher than $30 \mathrm{~dB}$ in a $2 \mathrm{GHz}$ bandwidth centered at $\mathrm{f}=12 \mathrm{GHz}(16.7 \%)$. In the inset, the electric field configuration is shown. forming the transducer are connected for a final optimization to fulfill the specifications of return loss level and high purity conversion. Although only one half of the converter is analyzed due to the symmetry plane, the high computational cost makes of critical importance to minimize the number of unknowns to optimize.

Moreover, the cost function has to include the attenuation of the four higher propagating modes in the circular waveguide $\left(\mathrm{TE}_{11 \mathrm{c}}, \mathrm{TE}_{21 \mathrm{c}}, \mathrm{TM}_{11 \mathrm{~s}}, \mathrm{TE}_{31 \mathrm{c}}\right.$ ) whose radius is a key optimization parameter for a double reason: first, to obtain a broadband return loss response with $30 \mathrm{~dB}$ level and second, to limit the number of propagating modes. The final structure of the transducer is presented in the inset of Fig. 4, where the electric field configuration is also shown. The simulated response with a return loss level higher than $30 \mathrm{~dB}$ and attenuation level higher than $50 \mathrm{~dB}$ for the four propagating modes in the design band from 11 to $13 \mathrm{GHz}$ can be seen in Figs. 4 and 5, respectively.

Considering the use of the transducer in applications of high-energy particle accelerators or plasma heating, its power rating has been calculated at the lowest frequency of operation $(11 \mathrm{GHz})$. Assuming a break down field of $30 \mathrm{kV} / \mathrm{cm}$ and the critical dimension in the feeding network, a $240 \mathrm{~kW}$ value has been obtained.

In order to verify the theoretical results, two similar transducers have been manufactured in brass. The insertion loss and the return loss have been measured in a back-toback configuration. The former is shown in Fig. 6 compared with the simulation, considering the conductivity of the brass $\sigma_{\text {brass }}=15.9 \times 10^{6}(\mathrm{~S} / \mathrm{m})$. The agreement is excellent, the insertion loss of the transducer being $0.075 \mathrm{~dB}$. In the inset, a CAD model of the dual structure is added for the sake of clarity. Figure 7 shows the comparison of the return loss considering the same conductivity value. The agreement is very good, being the average value less than $30 \mathrm{~dB}$. In its inset, a photo of the manufactured prototype placed in the measurement bench has been included.

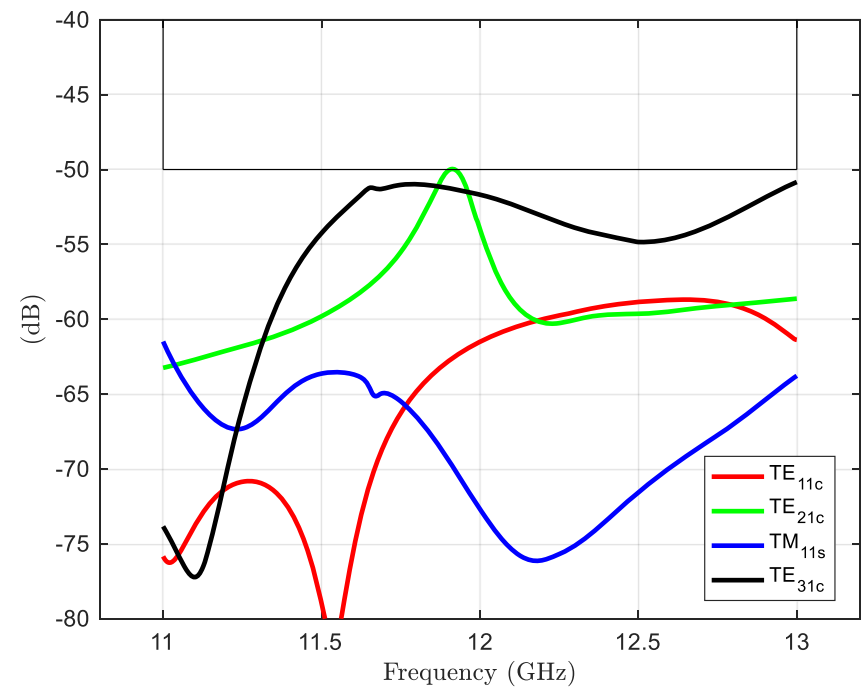

FIG. 5. Simulated attenuation level of the four propagation modes, higher than $50 \mathrm{~dB}$ in the $11-13 \mathrm{GHz}$ band. 


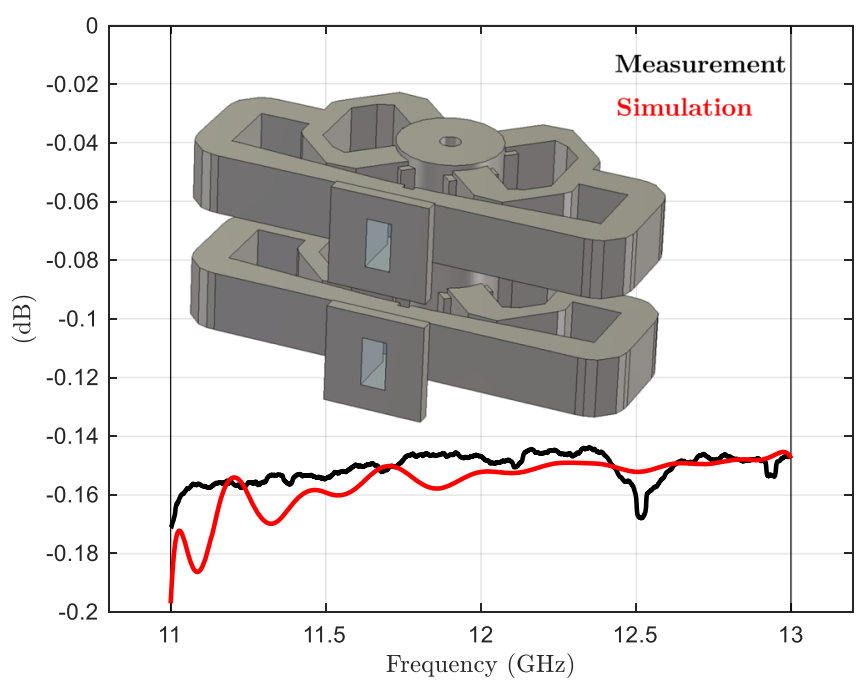

FIG. 6. Insertion loss comparison between the measurement and the simulation of the transducer in a back-to-back configuration, considering the conductivity of the brass $\sigma_{\text {brass }}=15.9 \times 10^{6}(\mathrm{~S} / \mathrm{m})$. In the inset, a 3D CAD view of the back-to-back is shown.

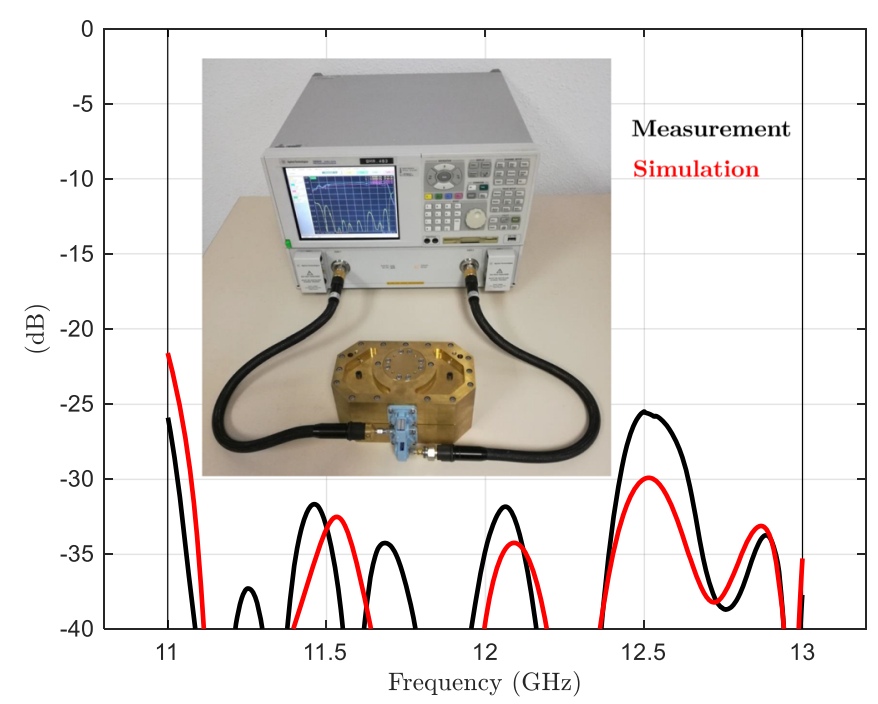

FIG. 7. Return loss comparison between the measurement and the simulation of the transducer in a back-to-back configuration, considering the conductivity of the brass $\sigma_{\text {brass }}=15.9 \times 10^{6}(\mathrm{~S} / \mathrm{m})$. In the inset, a photo in the measurement bench is shown.

\section{DESIGN OF THE 16-WAY POWER DIVIDER}

Once the transducer has been designed, the $\mathrm{TE}_{01}$ circular waveguide mode has to excite the divider to obtain the same response at the sixteen output ports. The signal entering the circular waveguide is equally divided in magnitude and phase. Each waveguide port must be very well matched, and the device should have low insertion loss over the whole operation bandwidth. A matching element, basically a post or cylinder that maintains the radial symmetry, is placed at the bottom of the structure. In this design, two stacked cylinders are necessary to cope with the bandwidth (see the inset in Fig. 9).

Usually, reduced height rectangular waveguides are connected to the divider base. ${ }^{11,12}$ By means of a stepped

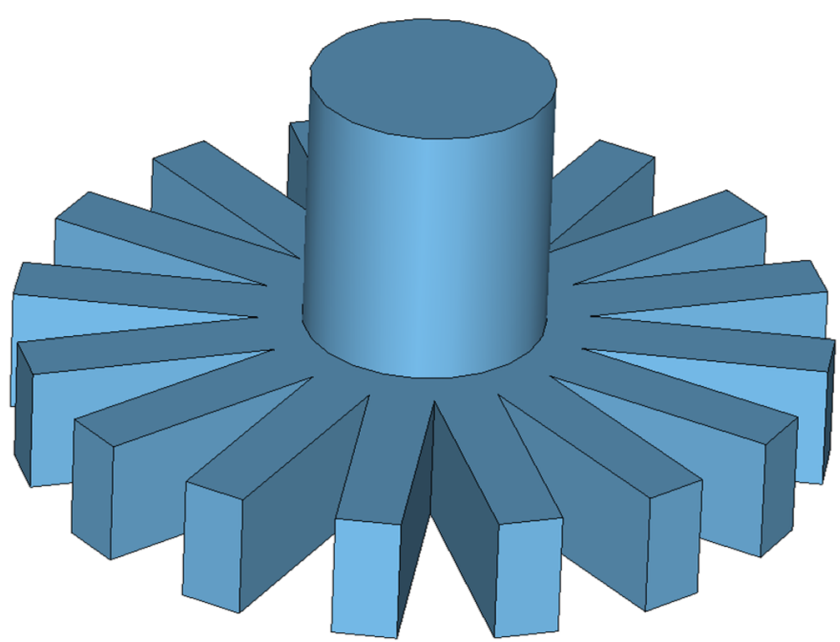

FIG. 8. 3D CAD view of the 16-way divider with the standard WR75 waveguides directly attached to the base without stepped impedance transformers.

impedance transformer, the standard height for the waveguide port is recovered. The drawback of this configuration is the increase in the insertion loss level and in the size. For this reason, in the design proposed here, the standard WR75 waveguides are directly attached to the base (Fig. 8). Therefore, even though 16 output ports are allocated, the radius of the base size is not enlarged, the losses are minimized, and the power handling is increased. In addition, the manufacturing process is simplified and the cost is reduced.

The radial symmetry with appropriate EW boundary conditions is exploited to reduce the computation time drastically. Figure 9 shows the theoretical full-wave simulated response of one quarter of the divider. The return loss level is better than $30 \mathrm{~dB}$ in the $2 \mathrm{GHz}$ bandwidth; the insertion loss in ports $\mathrm{P}_{3}$, $\mathrm{P}_{4}$, and $\mathrm{P}_{5}$ is $6 \mathrm{~dB}$ that corresponds to a 4-way power divider. In ports $\mathrm{P}_{2}$ and $\mathrm{P}_{6}$, with half-height, the level is $3 \mathrm{~dB}$ lower than in the other three, i.e., $9 \mathrm{~dB}$ (see the port numbering in

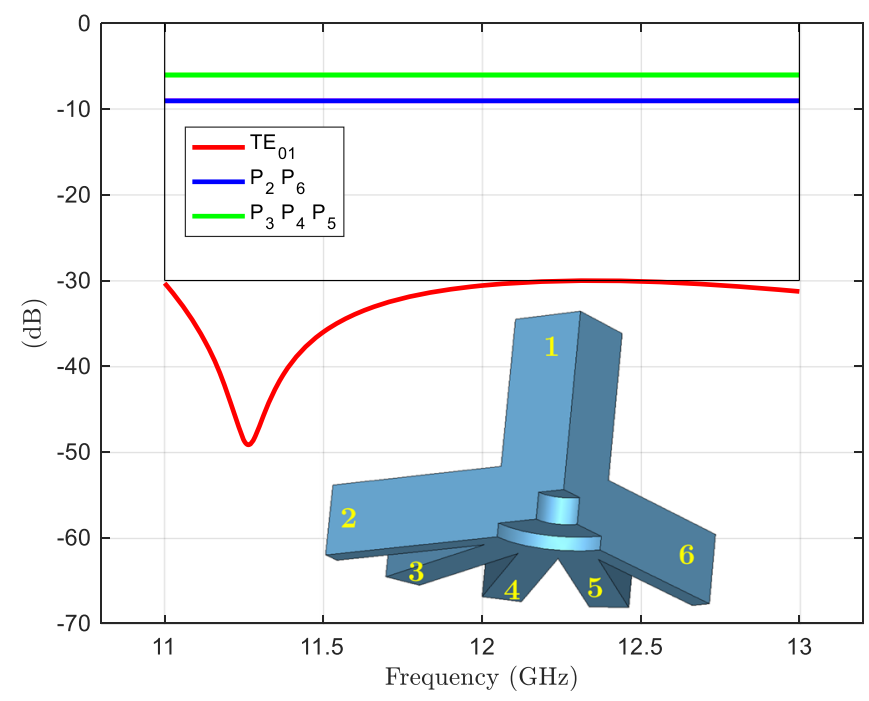

FIG. 9. Simulated response of the reflection coefficient of the $\mathrm{TE}_{01}$ mode, and the transmission to the rectangular ports, in the 16-way divider (only one quarter of the structure). In the inset, a 3D CAD view including the port numbering is shown. 
the inset of Fig. 9). After this response is obtained, the 16-way power divider is ready to be connected to the transducer.

\section{FINAL DESIGN OF THE COMBINER AND EXPERIMENTAL RESULTS}

As previously mentioned, the final step in the combiner design implies the connection of the transducer and the divider. Since the return loss level in both components has been carefully controlled, the final optimization is a simple task since only a few parameters are considered in the process: the radius of the circular waveguide and the dimensions of the three matching cylinders, two in the divider and one in the transducer (7 parameters).

A prototype of the 16-way radial power combiner has been manufactured in brass. The device has been mechanized in four parts, two halves corresponding to the transducer and another two halves corresponding to the divider, in both cases using the E-plane configuration to reduce the losses. Figure 10 shows the combiner during the experimental characterization of the scattering parameters with the high-precision WR75 matching loads.

In Fig. 11, the comparison between the simulation and the measurement of the return loss level is shown, under excitation by port 1 . It can be observed that the agreement is excellent. It is highlighted that the measured value is better than $30 \mathrm{~dB}$ in the 11-13 GHz bandwidth (except a slight deviation at the

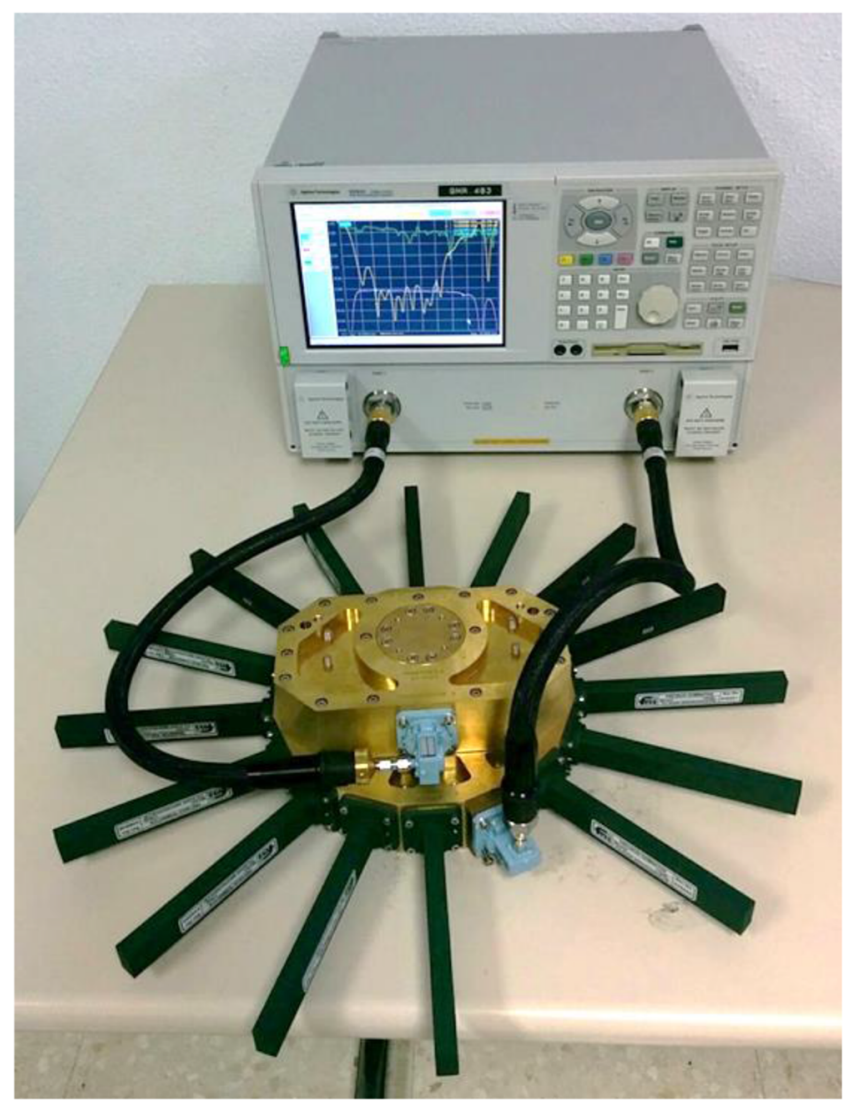

FIG. 10. Manufactured prototype in the measurement bench including the matching precision loads in the output ports for the experimental characterization.

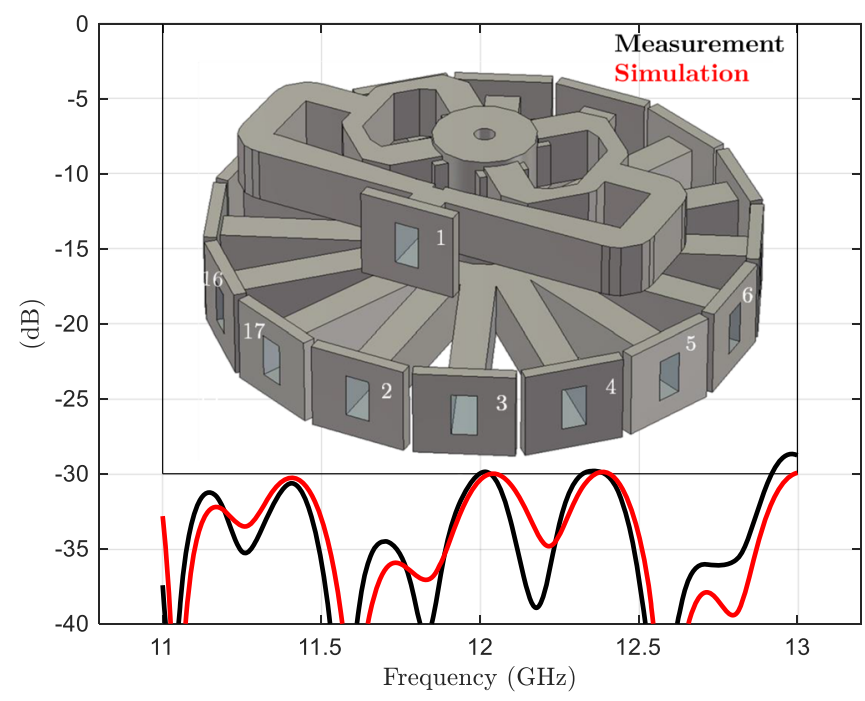

FIG. 11. Comparison between the simulation and the measurement corresponding to the return loss level of the power combiner excited in port 1 . In the inset, a 3D CAD view is shown including the port numbers.

end of the band). In the inset, the port numbering has been included in a 3D CAD view. Figure 12 presents the measured insertion loss of the 16-way combiner compared with the theoretic value assuming a perfect conductor $(\sigma=\infty,-12.04 \mathrm{~dB})$, and the average value simulated corresponding to the brass conductivity $(-12.15 \mathrm{~dB})$, showing that the effective conductivity for the manufacturing has virtually achieved the nominal value. The amplitude balance between the extreme values is $\pm 0.15 \mathrm{~dB}$, an outstanding result.

The phase response of the sixteen output ports is shown in Fig. 13. In the inset, it can be observed that the balance between the extreme values is $\pm 2.5^{\circ}$, again a very good result. Finally, Fig. 14 shows the isolation response corresponding to the excitation through a generic output port, the sixth in this case

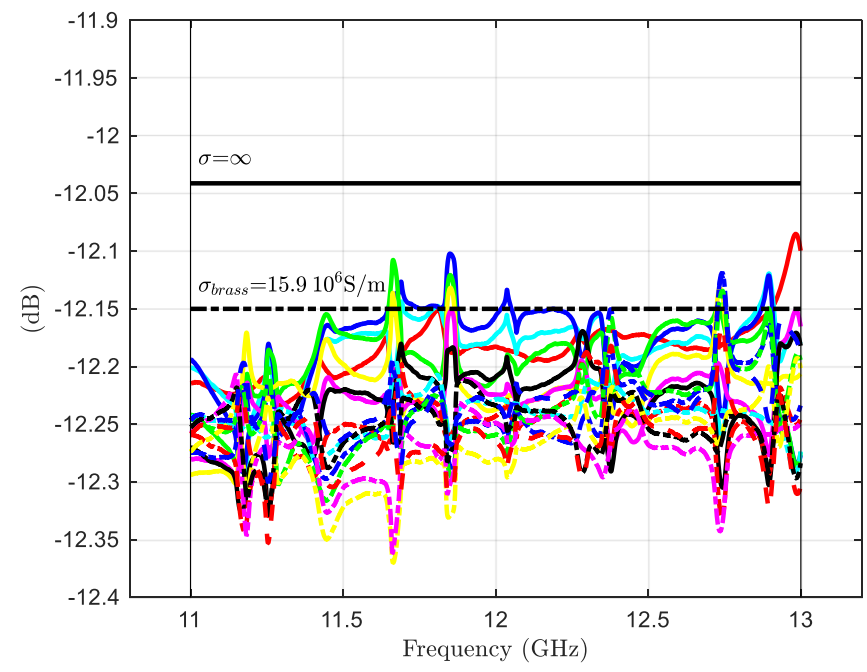

FIG. 12. Measured insertion loss of the 16-way combiner compared with the theoretic value assuming a perfect conductor $(\sigma=\infty,-12.04 \mathrm{~dB})$ and the average value simulated corresponding to the brass conductivity $\left[\sigma_{\text {brass }}=15.9\right.$ $\left.\times 10^{6}(\mathrm{~S} / \mathrm{m}),-12.15 \mathrm{~dB}\right]$. 


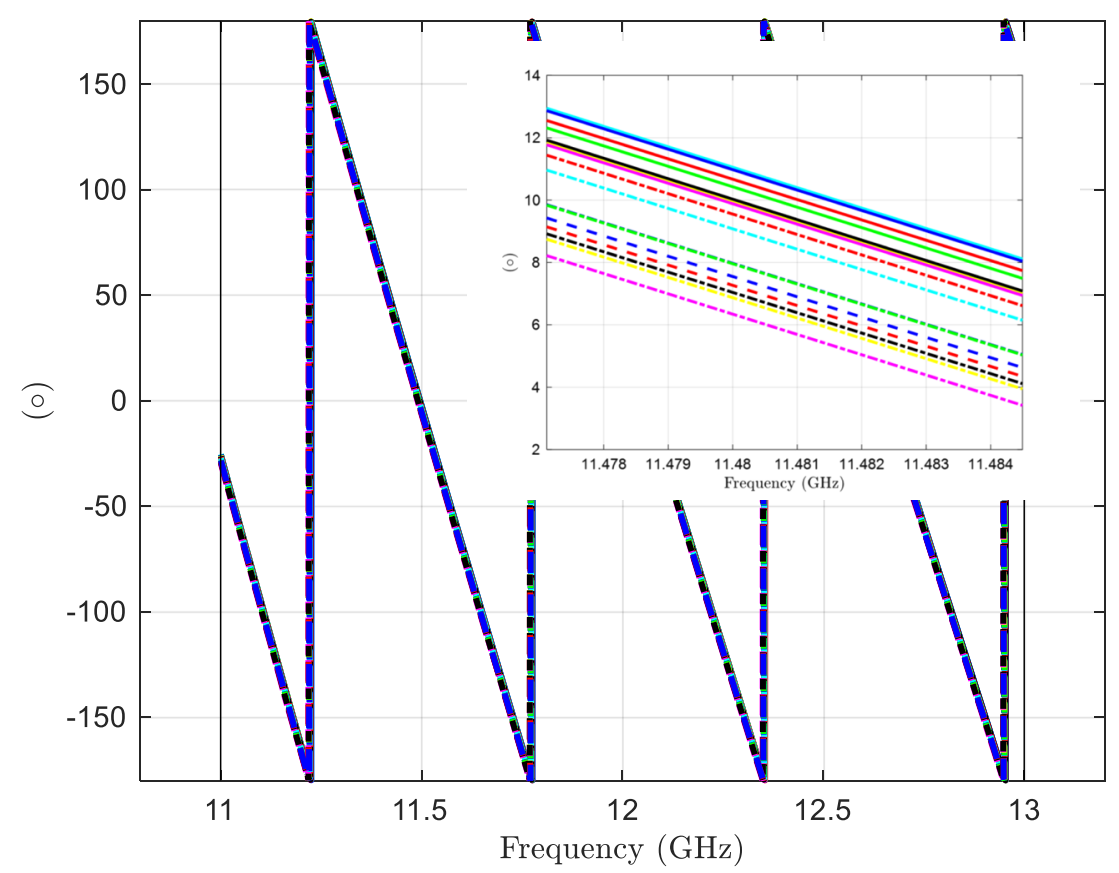

FIG. 13. Measured phases of the 16way combiner, with a detail in the inset.

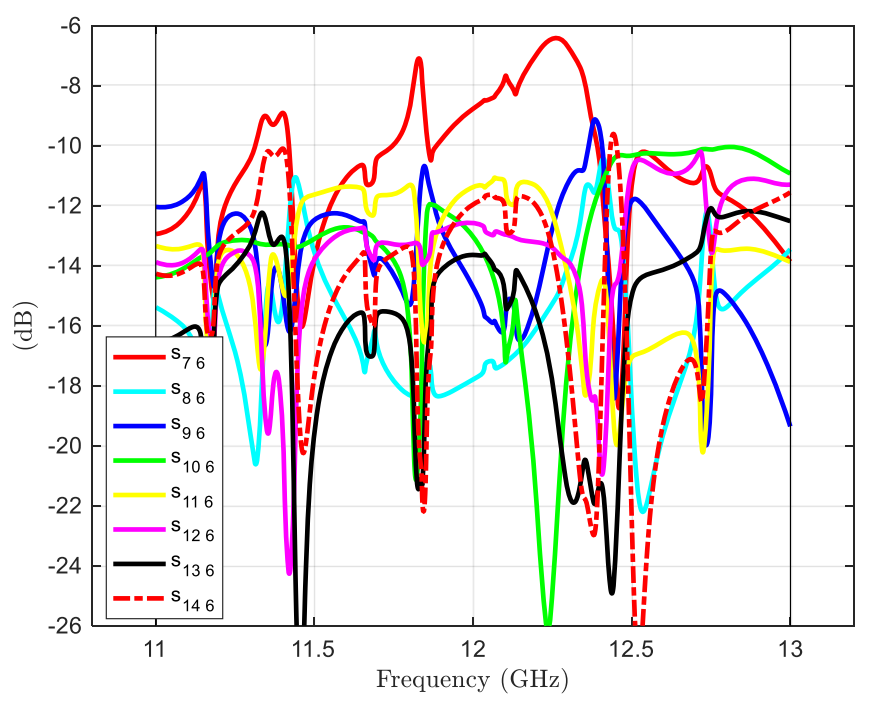

FIG. 14. Measured isolation response corresponding to the excitation through the sixth port (see the inset in Fig. 11), to the adjacent ports of the middle of the combiner, i.e., $S_{76}, S_{86}$, etc., until $S_{146}$ parameter.

(see the inset in Fig. 11), to the adjacent ports of the middle of the combiner, i.e., $S_{76}, S_{86}$, etc., until $S_{14} 6$ parameter. The average value, considering the eight responses, is approximately $-14 \mathrm{~dB}$. However, it is relevant to highlight that the worst isolation corresponds to the one between two contiguous ports, the $\mathrm{S}_{76}$ parameter in this case, where the minimum value is close to $-6 \mathrm{~dB}$.

\section{v. CONCLUSION}

A 16-way Ku band radial power combiner using the $\mathrm{TE}_{01}$ waveguide circular mode has been designed and measured for high-frequency low-loss systems found in amplification or plasma systems. A systematic step-by step process has been followed to control the partial responses of the two constituent devices making up the structure, i.e., the mode transducer and the radial divider.

In the case of the mode transducer, a rigorous analysis of the symmetry planes and mode families involved in the structure has led to a strict control of the modes in the problem. In the case of the divider, the output waveguides maintain the full height unlike the usual configuration, without increasing the radius of its base, being a relevant contribution. In all cases, the symmetry of every intermediate structure has been taken into account to reduce the computational effort in the optimization process. Moreover, the geometry has been simplified as much as possible to ease the manufacturing process and to reduce the cost.

The experimental results are, to the authors' knowledge, the state-of-the-art of radial Ku-band combiners based on the $\mathrm{TE}_{01}$ waveguide circular mode, considering the return loss, the insertion loss, and the phase and amplitude balance, for the 16-way non-reduced height standard ports achieved in a $16.7 \%$ fractional bandwidth.

\section{ACKNOWLEDGMENTS}

The authors would like to thank INMEPRE S.A., the diligence in the manufacturing process. This work was supported by the Spanish government under Grant (ADDMATE) No. TEC2016-76070-C3-1/2-R (AEI/FEDER/UE) and the program of Comunidad de Madrid S2013/ICE-3000 (SPADERADARCM).

\footnotetext{
${ }^{1}$ P. Khan, L. Epp, and A. Silva, "A Ka-band wide-bandgap solid-state power amplifier: Architecture performance estimates," IPN Progress Report 42164, Vol. 42, p. 163, 2005.

${ }^{2}$ K. Kanto, A. Satomi, Y. Asahi, Y. Kashiwabara, K. Matsushita, and K. Takagi, "An X-band $250 \mathrm{~W}$ solid-state power amplifier using $\mathrm{GaN}$ power HEMTs," in 2008 IEEE Radio and Wireless Symposium (IEEE, 2008), pp. $77-80$.
} 
${ }^{3}$ J. Schellenberg, E. Watkins, M. Micovic, B. Kim, and K. Han, "W-band, $5 \mathrm{~W}$ solid-state power amplifier/combiner," in IEEE MTT-S International Microwave Symposium Digest (MTT), 2010 (IEEE, 2010), pp. 240-243.

${ }^{4}$ J. X. Qiu et al., "Vacuum tube amplifiers," IEEE Microwave Mag. 10(7), 38-51 (2009)

${ }^{5}$ K. J. Russell, "Microwave power combining techniques," IEEE Trans. Microwave Theory Tech. 27(5), 472-478 (1979).

${ }^{6} \mathrm{~K}$. Chang and C. Sun, "Millimeter-wave power-combining techniques," IEEE Trans. Microwave Theory Tech. 31, 91-107 (1983).

${ }^{7}$ J. R. Montejo-Garai, C. A. Leal-Sevillano, J. A. Ruiz-Cruz, J. M. Rebollar, and T. Estrada, "Synthesis and design of waveguide band-stop filters without out-of-band spurious responses for plasma diagnosis," Fusion Eng. Des. 87(9), 1662-1666 (2012).

${ }^{8}$ Y. L. Chen, B. Lu, X. Y. Bai, J. Liang, C. Wang, H. Zeng, and J. Rao, "Design and measurement of a $3.7 \mathrm{GHz}$ high power recombiner," Fusion Eng. Des. 112, 232-235 (2016).

${ }^{9}$ T.-I. Hsu and M. D. Simonutti, "A wideband $60 \mathrm{GHz}$ 16-way power divider/combiner network," in IEEE MTT-S International Microwave Symposium Digest (IEEE, 1984), pp. 175-177.

${ }^{10} \mathrm{M}$. Chen, "A 19-way isolated power divider via the TE01 circular waveguide mode transition," in IEEE MTT-S International Microwave Symposium Digest, 1986 (IEEE, 1986), pp. 511-513.

${ }^{11}$ L. Epp, P. Khan, and A. Silva, "Ka-band wide-bandgap solid-state power amplifier: Hardware validation," IPN Progress Report 42-163, 2005.

${ }^{12}$ Q.-X. Chu, D.-Y. Mo, and Q.-S. Wu, "An isolated radial power divider via circular waveguide-mode transducer," IEEE Trans. Microwave Theory Tech. 63(12), 3988-3996 (2015).

${ }^{13}$ P. Khan and L. Epp, "Ka-band wide-bandgap solid-state power amplifier: Prototype combiner spurious mode suppression and power constraints," IPN Progress Report 42-163, 2006.

${ }^{14}$ J. R. Montejo-Garai, I. O. Saracho-Pantoja, J. A. Ruiz-Cruz, and J. M. Rebollar, "Broadband and high-purity Ku-band circular $\mathrm{TE}_{01}$-mode converter," in 2016 Asia-Pacific Microwave Conference (APMC) (IEEE, 2016), pp. 1-4.

${ }^{15} \mathrm{G}$. Southworth, Principles and Applications of Waveguide Transmission, The Bell Telephone Laboratories Series (Van Nostrand, 1956).
${ }^{16}$ C. A. Leal-Sevillano, J. A. Ruiz-Cruz, J. R. Montejo-Garai, and J. M. Rebollar, "Field propagation in circular hollow waveguides with non-ideal metallic conductors from microwaves to terahertz frequencies," IEEE Trans. Microwave Theory Tech. 59(12), 3013-3022 (2011).

${ }^{17}$ K. Chu, "The electron cyclotron maser," Rev. Mod. Phys. 76(2), 489 (2004).

${ }^{18}$ M. Blank, B. G. Danly, and B. Levush, "Experimental demonstration of a W-band (94 GHz) gyrotron amplifier," IEEE Trans. Plasma Sci. 27(2), 405-411 (1999).

${ }^{19}$ K. Leou, D. McDermott, A. Balkcum, and N. Luhmann, "Stable high-power $\mathrm{TE}_{01}$ gyro-TWT amplifiers," IEEE Trans. Plasma Sci. 22(5), 585-592 (1994).

${ }^{20}$ M. Garven, J. P. Calame, B. G. Danly, K. T. Nguyen, B. Levush, F. Wood, and D. E. Pershing, "A gyrotron-traveling-wave tube amplifier experiment with a ceramic loaded interaction region," IEEE Trans. Plasma Sci. 30(3), $885-893$ (2002).

${ }^{21} \mathrm{P}$. Wolfert, "A wide-band rectangular-to-circular mode transducer for millimetre waves (correspondence)," IEEE Trans. Microwave Theory Tech. 11(5), 430-431 (1963).

${ }^{22}$ G. Marie, British patent 805519 (December 1958).

${ }^{23}$ S. Saad, J. Davies, and O. Davies, "Analysis and design of a circular $\mathrm{TE}_{01}$ mode transducer," IEE J. Microwaves, Opt. Acoust. 1(2), 58-62 (1977).

${ }^{24}$ Q.-Z. Xue, S.-C. Zhang, and P.-K. Liu, "Design of the $\mathrm{H}_{10}$ to $\mathrm{H}_{01}$ sectortype mode converter at Ka-band," Int. J. Infrared Millimeter Waves 26(10), 1407-1415 (2005).

${ }^{25} \mathrm{G}$. Nusinovich, R. Barker, N. Luhmann, and J. Booske, in Modern Microwave and Millimetre-Wave Power Electronics (IEEE Press, 2005), Chap. 11.

${ }^{26}$ H. Hoag, S. Tantawi, R. Callin, H. Deruyter, Z. Farkas, K. Ko, N. Kroll, T. Lavine, A. Menegat, and A. Vlieks, "Flower-petal mode converter for NLC," in Proceedings of the IEEE Particle Accelerator Conference, 1993 (IEEE, 1993), pp. 1121-1123.

${ }^{27}$ C.-F. Yu and T.-H. Chang, "High-performance circular $\mathrm{TE}_{01}$-mode converter," IEEE Trans. Microwave Theory Tech. 53(12), 3794-3798 (2005).

${ }^{28}$ See https://www.cst.com/ for CST, Computer Simulation Technology. 